TRANSACTIONS OF THE

AMERICAN MA THEMATICAL SOCIETY

Volume 184, October 1973

\title{
PRIME IDEALS AND SHEAF REPRESENTATION OF A PSEUDO SYMMETRIC RING
}

BY

GOOYONG SHIN

\begin{abstract}
Almost symmetric rings and pseudo symmetric rings are introduced. The classes of symmetric rings, of almost symmetric rings, and of pseudo symmetric rings are in a strictly increasing order. A sheaf representation is obtained for pseudo symmetric rings, similar to the cases of symmetric rings, semiprime rings, and strongly harmonic rings. Minimal prime ideals of a pseudo symmetric ring have the same characterization, due to J. Kist, as for the commutative case. A characterization is obtained for a pseudo symmetric ring with a certain right quotient ring to have compact minimal prime ideal space, extending a result due to Mewborn.
\end{abstract}

Introduction. Recently Koh [9] has obtained a sheaf representation of a ring without nilpotent elements. While Lambek [12] has unified this and the commutative case by introducing symmetric rings, Hofmann [7, Theorems 1.17 and 1.24] has extended the representation to semiprime rings. Using the maximal modular ideal space, Koh [11] has also obtained the representation for strongly harmonic rings.

In this paper, the result of Lambek [12] is extended to a larger class of ringspseudo symmetric rings (Theorem 3.5). Example $5.1(\mathrm{e})$ is an example of a pseudo symmetric ring whose representation does not fall under any other types mentioned above. (See [7, p. 311].) Almost symmetric rings are also introduced. A symmetric ring is almost symmetric and an almost symmetric ring is pseudo symmetric, but not conversely in either case. Some properties of these rings are discussed in the first two sections.

In pseudo symmetric rings, the minimal prime ideals have the same characterization as for the commutative case. Mewborn's characterization of a commutative ring with compact minimal prime ideal space is generalized to pseudo symmetric rings with certain right quotient ring. For a pseudo symmetric ring, its prime ideal space is a $T_{1}$-space iff it is a completely regular $T_{2}$-space iff its usual basic open sets are closed as well.

Presented to the Society, November 24, 1972 and January 28, 1973 under the title Prime ideal space and sheaf representation; received by the editors October 16, 1972. AMS (MOS) subject classifications (1970). Primary 16A64, 16A66, 18F20, 16A34; Secondary 16A48, 54D10, 54D20, 54H10.

Key words and phrases. Prime ideal, sheaf representation, pseudo symmetric ring, minimal prime ideal space. 
Acknowledgment. This paper is based on the author's doctoral dissertation at the North Carolina State University. The author wishes to express his sincere appreciation to his thesis advisor Professor Kwangil Koh; to Professor Jiang Luh for the idea of Example 5.1; and to the referee for many helpful suggestions and comments. In particular, Example 5.6 belongs to the referee.

1. Almost symmetric rings and pseudo symmetric rings. Throughout this paper a ring is an associative ring which need not have an identity. Lambek [12] calls a ring $R$ symmetric provided $a b c=0$ implies $a c b=0$ for any $a, b, c \in R$.

Lemma 1.1 (Lambek). A ring without (nonzero) nilpotent elements is symmetric.

Proof. If $a b c=0$, then $c(a b c) a b=0$ and $c a b=0$. Then $a b a(c a b) a c=0$, $a b a c=0, b a c b(a b a c) b a=0, b a c b a=0, a c(b a c b a) c b=0$, hence $a c b=0$.

We shall call a ring $R$ almost symmetric if it satisfies:

(S I) For each element $a \in R, a^{r}$ is an ideal of $R$, where $a^{r}=\{b \in R: a b=0\}$; and

(S II) For any $a, b, c \in R$, if $a(b c)^{n}=0$ for a positive integer $n$, then $a b^{m} c^{m}=0$ for some positive integer $m$.

The following two lemmas are proved easily.

Lemma 1.2. For any ring $R$ the following are equivalent:

(a) $R$ satisfies (S I).

(b) Any annibilator right ideal of $R$ is an ideal of $R$.

(c) Any annibilator left ideal of $R$ is an ideal of $R$.

(d) For any $a, b \in R, a b=0$ implies $a R b=0$.

Lemma 1.3. If a ring $R$ satisfies (S I), then it satisfies (S II) iff for any $a, b$, $c \in R, a(b c)^{2}=0$ implies $a b^{m} c^{m}=0$ for a positive integer $m$.

Proposition 1.4. Any symmetric ring is almost symmetric.

Proof. Clearly any symmetric ring satisfies the condition 1.2(d). If $(a b) c(b c)=0$, then $(a b)(b c) c=0$.

An almost symmetric ring need not be symmetric. (See 5.1(a).) The conditions (S I) and (S II) are independent of each other. (See 5.1(c) and 5.2(b).) The prime radical $\operatorname{rad} R$ of a ring $R$ coincides with the set of all nilpotent elements of $R$ if $R$ is commutative. This is also true if $R$ is symmetric [12, Proposition 3].

Theorem 1.5. If a ring $R$ satisfies (S I) then its prime radical coincides with the set of all nilpotent elements of $R$.

Proof. It suffices to show that $\operatorname{rad} R$ contains all the nilpotent elements of $R$ because any element of $\operatorname{rad} R$ is always nilpotent. Suppose $a^{n}=0$. If $a \notin P$ for some prime ideal $P$ then $a x_{1} a \notin P$ for some element $x_{1}$ of $R$. Continuing the 
process we can find elements $x_{i}$ of $R$ such that $P$ does not contain $b=a x_{1} a \cdots$ $x_{n-1} a$. But, by (S I), $a^{n}=0$ implies $b=0$, hence $b \in P$, a contradiction.

We shall call a ring $R$ pseudo symmetric if it satisfies:

(PS I) The prime radical $\operatorname{rad}(R / I)$ is the set of all nilpotent elements of the ring $R / I$, whenever $I=(0)$ or $I$ is the right annihilator of $a R$ in $R$ for some $a \in R$; and

(PS II) For any $a, b, c \in R$, if $a R(b c)^{n}=0$ for a positive integer $n$, then $a(R b R)^{m} c^{m}=0$ for some positive integer $m$.

Proposition 1.6. Let $R$ be a ring with (S I). Then

(a) $R$ satisfies (PS I); and

(b) $R$ satisfies (PS II) iff it satisfies (S II).

In particular an almost symmetric ring is pseudo symmetric.

Proof. Let $A$ be any subset of $R$. For each $a \in A$, if $a b c=0$ then $a b R c=0$. Thus $R / A^{r}$ has (S I) for any subset $A$ of $R$, if $R$ has (S I). This proves (a) by 1.5. For (b), suppose that $R$ satisfies (S II). If $a R(b c)^{n}=0$, then $a(b c)^{n+1}=0$, $a b^{m} c^{m}=0$ by (S II), hence $a(R b R)^{m} c^{m}=0$ by (S I). Conversely suppose that $R$ has (PS II). If $a(b c)^{n}=0$, then $a R(b c)^{n}=0$ by (S I), $a(R b R)^{m} c^{m}=0$ by (PS II) and $a b^{3 m} c^{3 m}=0$, completing the proof.

A pseudo symmetric ring need not be almost symmetric. (See 5.1(c).) Hence according to 1.4 and 1.6 the classes of symmetric rings, of almost symmetric rings, and of pseudo symmetric rings are in a strictly increasing order.

The following proposition shows that in semiprime rings (PS I), (S I), 'almost symmetric', 'pseudo symmetric' and 'symmetric' are equivalent concepts.

Proposition 1.7. For any ring $R$ the following are equivalent:

(a) $R$ bas no nilpotent elements,

(b) $R$ is a semiprime ring with (PS I).

Proof. Clear.

For a prime ideal $P$ of a ring $R$, let

$O(P)=\{a \in R: a R b=0$ for some $b \in R \backslash P\}$,

$O_{P}=\{a \in R: a b=0$ for some $b \in R \backslash P\}$,

$N(P)=\{a \in R: a R b \subseteq \operatorname{rad} R$ for some $b \in R \backslash P\}$,

$N_{P}=\{a \in R: a b \in \operatorname{rad} R$ for some $b \in R \backslash P\}$.

It is clear that $O(P)$ and $N(P)$ are ideals of $R$ contained in $O_{P}$ and $N_{P}$, respectively, and that $O(P)$ and $N(P)$ are subsets of $P$. If $R$ has the property $(S I)$ then $O(P)=O_{P}$. The proof of the following theorem is an adaptation from $[9$, Theorem 2.4].

Theorem 1.8. Let $R$ be a ring without nilpotent elements. For eacb $P \in \operatorname{Spec} R$, 


$$
O(P)=\bigcap\{Q \in \operatorname{Spec} R: O(P) \subseteq Q\}=\bigcap\{Q \in \operatorname{Spec} R: Q \subseteq P\}
$$

where $\operatorname{Spec} R$ is the set of all prime ideals of $R$.

Proof. If $Q \subseteq P$ then $O(P) \subseteq O(Q) \subseteq Q$. So we have

$$
O(P) \subseteq \cap\{Q: O(P) \subseteq Q\} \subseteq \bigcap\{Q: Q \subseteq P\}
$$

Suppose $a \notin O(P)$. We shall find a prime ideal $Q$ such that $a \notin Q$ and $Q \subseteq P$. The set $S=\left\{a, a^{2}, a^{3}, \cdots\right\}$ is a multiplicative system that does not contain 0 and $L=R \backslash P$ is an $m$-system. Let $T$ be the set of all nonzero elements of $R$ of the form $a^{t} x_{1} a^{t} x_{2} \cdots x_{n} a^{t} n$, where $x_{i} \in L$ and $t_{i}$ 's are positive integers with $t_{0}$ and $t_{n}$ allowed to be zero. Let $M=S \cup T$. Note that $L \subseteq T$. We claim that xay $\in M$ for any $x, y \in M$. If $x, y \in S$ then $x a y \in S$. Let $x \in S, y \in T$ with $x=a^{s}, y=$ $a^{t} x_{1} a^{t} x_{2} \cdots x_{n} a^{t}{ }^{n}$. If $x a y \neq 0$, then $x a y \in T$. Since $x_{i} \in L, w=x_{1} z_{1} \cdots$

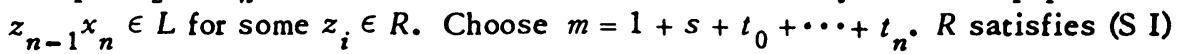
by 1.1. If $x a y=0$ then by (S I), $(a w)^{m}=0$ and $a w=0$, hence $a \in O_{P}=O(P)$, a contradiction. Similarly one shows that if $x, y \in T$ then $x a y \neq 0$ and $x a y \in T$. This shows $M$ is an m-system that is disjoint from (0), hence there is a prime ideal $Q$ that is disjoint from $M$. Then $a \notin Q$ and $Q \subseteq P$, completing the proof.

Immediately from the theorem we have the following corollary, which is a partial answer to a question raised by Gillman [3, Theorem 2.6].

Corollary 1.9. If $\operatorname{rad} R$ coincides with the set of all nilpotent elements of $R$, then for each $P \in \operatorname{Spec} R$, $N(P)=\bigcap\{Q \in \operatorname{Spec} R: N(P) \subseteq Q\}=\bigcap\{Q \in \operatorname{Spec} R: Q \subseteq P\}$.

The following has been obtained by Kist [8] for commutative rings, and by Koh $[9,2.4]$ for rings without nilpotent elements. For (a) $\Leftrightarrow$ (b) see also Hofmann $[7,1.33]$.

Corollary 1.10. If $\mathrm{rad} R$ coincides with the set of all nilpotent elements of $R$, then for each $P \in \operatorname{Spec} R$ the following are equivalent:

(a) $P$ is a minimal prime deal.

(b) $N(P)=P$.

(c) For any a $\in P$, ab is nilpotent for some $b \in R \backslash P$.

Proof. (a) $\Leftrightarrow$ (b) follows from 1.9. (b) $\Rightarrow$ (c): For each $a \in P=N(P)$, $a b a b \in a R b \subseteq \operatorname{rad} R$ for some $b \in R \backslash P$, hence $a b$ is nilpotent. (c) $\Rightarrow$ (b): If $a \in P$ and $a b \in \operatorname{rad} R$ for some $b \in R \backslash P$, then $a R b \subseteq \operatorname{rad} R$ because the $\operatorname{ring} R / \mathrm{rad} R$ satisfies (S I) by 1.1. Hence $a \in N(P)$, and $N(P)=P$ since $N(P) \subseteq P$ always. 
Proposition 1.11. For any ring $R$, the following are equivalent:

(a) $\operatorname{rad} R$ coincides with the set of all nilpotent elements of $R$.

(b) Every minimal prime ideal is completely prime.

Proof. (b) $\Rightarrow$ (a) is immediate since $\operatorname{rad} R$ is the intersection of all minimal prime ideals. (a) $\Rightarrow$ (b): Let $P$ be a minimal prime ideal of $R$ such that $a b \in P$. By $1.10 a b c \in \operatorname{rad} R$ for some $c \in R \backslash P$. If $b \notin P$ then $b z c \notin P$ for some $z \in R$. By (S I) of $R / \mathrm{rad} R, a R b z c \subseteq \operatorname{rad} R$ and $a \in N(P)=P$.

Corollary 1.12. If $\mathrm{rad} R$ coincides with the set of all nilpotent elements of $R$, then $N(P)=N_{P}$ for each prime ideal $P$ of $R$.

Proposition 1.13. For any ring $R$ the following are equivalent:

(a) Every prime ideal of $R$ is completely prime.

(b) For any ideal $I$ of $R, \operatorname{rad}(R / I)$ coincides with the set of all nilpotent ele. ments of $R / l$.

Proof. (a) $\Rightarrow$ (b): If $a^{n} \in I \subseteq P$ then $a \in P$, since $P$ is completely prime. (b) $\Rightarrow$ (a): If $P$ is a prime ideal of $R, R / P$ is a prime ring, and by (b) and 1.11 the minimal prime ideal of $R / P$ is completely prime. Then $P$ must be completely prime.

Proposition 1.14. Let $R$ be a ring with (S I). Then $R$ satisfies (S II), if either

(a) $O(P)=0$ for every prime ideal $P$ of $R$; or

(b) $P \cap Q$ is a prime ideal for any prime ideals $P$ and $Q$ of $R$.

Proof. Let $a(b c)^{2}=0$. Assume (a). If either $b \in \operatorname{rad} R$ or $c \in \operatorname{rad} R$, then either $b$ or $c$ is nilpotent and $a b^{m} c^{m}=0$. Suppose $b \notin P$ and $c \notin Q$ for some prime ideals $P$ and $Q$ of $R$. Then $a b c b \in O_{Q}=O(Q)=0, a b c \in O(P)=0$, finally $a \in O(P)$ and $a=0$. Now assume (b). If either $b+a^{r} \epsilon \operatorname{rad}\left(R / a^{r}\right)$ or $c+a^{r} \in \operatorname{rad}\left(R / a^{r}\right)$, then either $a b^{m}=0$ or $a c^{m}=0$, hence $a b^{m} c^{m}=0$ by (S I). If there are prime ideals $P$ and $Q$ of $R$ such that $b \notin P, c \notin Q$ and $a^{r} \subseteq P \cap Q$, then since $P \cap Q$ is a prime ideal, $d=b u c v b w c \notin P \cap Q$. From $(\mathrm{S} \mathrm{I})$ and $a(b c)^{2}=0$, it follows that $a d=0$, hence $a^{r} \underline{\unrhd} P \cap Q$, a contradiction.

The following follows from 1.11 .

Proposition 1.15. A ring is an integral domain iff it is a prime ring with (PS I).

Proposition 1.16. Let $R$ be a (von Neumann) regular ring. Then the following are equivalent:

(a) $R$ satisfies (PS I),

(b) $R$ is strongly regular,

(c) every idempotent of $R$ is central.

Proof. A regular ring is semiprime and it is strongly regular iff it has no 
nilpotent elements. Then (a) $\Leftrightarrow(b)$ follows from 1.7. Any idempotent element of a ring without nilpotents is central, and $(b) \Rightarrow(c)$ follows. (c) $\Rightarrow(b)$ is trivial.

Theorem 1.17. For any ring $R$ the following are equivalent:

(a) $R$ is a (von Neumann) regular prime ring with (PS I),

(b) $R$ is a division ring.

Proof. (b) $\Rightarrow$ (a) is immediate. (a) $\Rightarrow$ (b): By $1.15, R$ is a regular integral domain. Then $R$ has a unique nonzero idempotent, because if $e^{2}=e \neq 0$ and $f^{2}=f \neq 0$ then $e(e f-f)=0,(e f-e) f=0$, hence $e=e f=f$. Then $R$ must be a division ring.

It is well known that there is a simple integral domain which is not a division ring. This shows that the regularity of $R$ cannot be dropped from 1.17(a).

2. Transfer theorems.

Theorem 2.1. If $R$ is an almost symmetric ring then $R / \mathrm{rad} R, R / N(P)$, $R / O(P)$ and $R / a^{r}$ are almost symmetric for eacb $P \in \operatorname{Spec} R$ and eacb $a \in R$.

Proof. (a) If $\operatorname{rad} R$ is the set of all nilpotents, then $R / \mathrm{rad} R$ and $R / N(P)$ have no nilpotent elements, for if $a^{n} \in N(P)$ with $a^{n} R c \subseteq \operatorname{rad} R$ for some $c \in R \backslash P$, then $a R c \subseteq \operatorname{rad} R$ and $a \in N(P)$ because every minimal prime ideal of $R$ is completely prime.

(b) Let $R$ satisfy (S I). $R / A^{r}$ and $R / A^{l}$ satisfy (S I) for any $A \subseteq R$, as shown in the proof of 1.6. $R / O(P)$ satisfies (S I), for if $a b \in O(P)$ with $a b R c=0$ for some $c \notin P$ then $a R b R c=0$ and $a R b \subseteq O(P)$.

(c) Let $R$ be almost symmetric. If $b(c d)^{2} \in O(P)$ with $b(c d)^{2} R f=0$ for some $f \notin P$ then $b(c d f)^{3}=0, b c^{n}(d f)^{n}=0, b c^{m} d^{m} f^{m}=0$ and $b c^{m} d^{m} R w=0$, where $w=$ $f v_{1} f v_{2} \cdots v_{m-1} f \notin P$. It shows that $R / O(P)$ satisfies (S II). If $a b(c d)^{2}=0$ then $a b c^{m} d^{m}=0$, thus $R / a^{r}$ satisfies (S II).

There exists an integral domain whose homomorphic image fails to satisfy (PS I). (See 5.3.)

Proposition 2.2. If a ring $R$ satisfies (S I) (resp. (S II)) then so does any subring of $R$.

Proof. Clear.

It is not clear whether a subring of a pseudo symmetric ring is necessarily pseudo symmetric.

Theorem 2.3. (a) The direct product $\Pi R_{a}$ satisfies (S I) iff each ring $R_{a}$ does. (b) If the direct product $\Pi R_{a}$ satisfies (S II) then so does each $R_{a^{*}}$

Proof. Each ring $R_{a}$ may be considered as a subring of the direct product. 
(b) and one part of (a) follow from 2.2. If each $R_{a}$ satisfies (S I) then it is clear that the ir direct product satisfies (S I).

Corollary 2.4. (a) If each $R_{a}$ satisfies (S I) then so does every subdirect sum of these rings $R_{\alpha}$.

(b) A direct sum $\Sigma^{\oplus} R_{a}$ is almost symmetric iff each $R_{\alpha}$ is almost symmetric.

Proof. If each $R_{a}$ satisfies (S I) then any subdirect sum, being a subring of the direct product, satisfies (S I) by 2.3(a). In this case the direct sum, being a subdirect sum, also satisfies (S I). If each $R_{a}$ is almost symmetric, then clearly their direct sum satisfies (S II). If the direct sum is almost symmetric then each $R_{a}$, being a subring of the direct sum, is almost symmetric by 2.2 .

Theorem 2.5. (a) If the direct product of rings $R_{a}$ satisfies (PS I), then so does each $R_{a^{*}}$.

(b) If the direct product of rings $R_{a}$ satisfies (PS II), then so does each $R_{\alpha}$.

Proof. Let $R$ be the direct product and let $\phi_{\beta}: R \rightarrow R_{\beta}$ be the projection map. If $a \in R_{\beta}$ and $P$ is a prime ideal of $R_{\beta}$ let $\bar{a} \in R$ such that $\phi_{\beta}(\bar{a})=a$ and $\phi_{a}(\bar{a})=0$ for all $a \neq \beta$, and let $\bar{P}$ be the inverse image of $P$ by $\phi_{\beta}$. Then $\bar{P}$ is a prime ideal of $R$. If $a$ is nilpotent $\bar{a}$ is also nilpotent. Then $\bar{a} \epsilon \bar{P}$ and $a \in P$. Now suppose $b^{n} \in I \subseteq P$, where $I$ is a right annihilator of $a R_{\beta}$ in $R_{\beta}$ and $a, b \in R_{\beta}$. Then $\bar{b}^{n} \in \bar{I} \subseteq \bar{P}$ where $\bar{I}$ is the inverse image of $I$ by $\phi_{\beta}$. This means $\bar{b} \in \bar{P}$ and $b \in P$, proving (a). (b) is clear.

Theorem 2.6. (a) If the direct sum of rings $R_{a}$ satisfies (PS I) then so does eacb $R_{a}$.

(b) The direct sum of rings $R_{\alpha}$ satisfies (PS II) iff so does each $R_{\alpha}$.

Proof. (a) is proved in a same manner as for 2.5(a). (b) is clear.

The converses of 2.3(b) and 2.5(b) are not true. (See 5.2(b) and (c).) If a subdirect sum of rings $R_{a}$ satisfies (S I) the rings $R_{a}$ need not satisfy (S I). (See 5.3.) It is not clear whether the converses of 2.5(a) and 2.6(a) are true.

Lemma 2.7. Let $R$ be a ring with identity. If $R$ satisfies (S I) then every idempotent of $R$ is central.

Proof. If $e^{2}=e$, then $e(1-e)=(1-e) e=0$ and $e a(1-e)=(1-e) a e=0$ for each $a \in R$. Then $e a=e a e=a e$.

If $R$ does not have an identity 2.7 need not be true. (See 5.4(b).) The converse of 2.7 need not be true. (See 5.5.) Let $R^{*}$ be the ring obtained by adjoining an identity to a ring $R$ in the usual manner. If $R$ satisfies (S I) then $R^{*}$ need not satisfy (S I). (See 5.4(d).) 
3. Sheaf representation. For a ring $R$, let $\operatorname{Spec} R$ be the set of all prime ideals of $R$, for any subset $A$ of $R$ let supp $A$ be the set of all prime ideals $P$ such that $A \nsubseteq P$, and let hull $A$ be the complement of $\operatorname{supp} A$ in $\operatorname{Spec} R$. In case $A=\{a\}$ we shall write supp $a$ and hull $a$.

Lemma 3.1. For any ring $R$, \{supp $a: a \in R\}$ is a base (for open sets) on Spec $R$. This topology is called the bull-kernel topology.

Proof. For any $P \in \operatorname{Spec} R, P \neq R$ and there is $a \in R \backslash P$. Thus the family covers Spec $R$. Suppose $P \in \operatorname{supp} a \cap \operatorname{supp} b$. Then $d=a c b \notin P$ for some $c \in R$, and $P \in$ supp $d \subseteq$ supp $a \cap \operatorname{supp} b$.

Lemma 3.2. If a ring $R$ bas an identity, then $\operatorname{Spec} R$ is a compact space.

Proof. See [2, p. 76].

Proposition 3.3. For a ring $R$, let $k(R)=\bigcup R / O(P)$ be the disjoint union of rings $R / O(P)$ with $P \in \operatorname{Spec} R$. For eacb $r \in R$, let $\hat{r}: \operatorname{Spec} R \rightarrow R(R)$ be defined as $\hat{r}(P)=r+O(P)$. Then $k(R)$ is a sheaf of rings over Spec $R$ with the topology on $k(R)$ generated by a base $\{\hat{r}(\operatorname{supp} a): a, r \in R\}$; and $\hat{r}$ is a global section for each $r \in R$.

Proof. See [7, p. 305]. For definitions, see [14, 3.1].

Let $\Gamma($ Spec $R, f(R))$ be the set of all global sections. Then this becomes a ring. The map $r \rightarrow \hat{r}$ is a ring homomorphism, called the Gelfand homomorphism, with its kernel $\cap O(P)$. Let $R$ have an identity. Recently Koh [9] has shown that this map is an isomorphism in the case when $R$ has no nilpotents, Lambek [12] for symmetric rings, Koh [11] for strongly harmonic rings, and Hofmann [7, 1.17] for semiprime rings.

Lemma 3.4. For any ring $R$, the Gelfand homomorphism is a monomorphism iff, for any $0 \neq a \in R,(a R)^{r}$ is contained in a prime ideal of $R$.

Proof. Clear from the definition of $O(P)$.

Theorem 3.5. Let $R$ be a pseudo symmetric ring with identity. Then $R$ is isomorphic onto $\Gamma(\operatorname{Spec} R, k(R))$, with $k(R)=\bigcup R / O(P)$.

Proof. If $a \neq 0$, then $(a R) R \neq 0$ since $1 \in R$. Hence $(a R)^{r}$ is contained in a maximal ideal. By the lemma the Gelfand homomorphism is a monomorphism. Let $\sigma$ be a global section. For $P \in \operatorname{Spec} R, \sigma(P)=\hat{a}(P)$ for some $a \in R$. By [14, 3.2] $\sigma$ and $\hat{a}$ agree on a neighborhood of $P$, hence on a basic open neighborhood supp $b$. By compactness of $\operatorname{Spec} R$, there are $a_{i}, b_{i} \in R$ such that $\operatorname{Spec} R=\bigcup_{i=1}^{n} \operatorname{supp} b_{i}$ and $\sigma(P)=\hat{a}_{i}(P)$ for each $P \in \operatorname{supp} b_{i}$. If $\left(\left(a_{i}-a_{j}\right) R\right)^{r} \subseteq P$, then $a_{i}-a_{j} \notin O(P)$, $\hat{a}_{i}(P) \neq \hat{a}_{j}(P)$, and $P \notin \operatorname{supp} b_{i} \cap \operatorname{supp} b_{j}$, hence $b_{i} b_{j} \in P$. This means that 
$b_{i} b_{j}+I \epsilon \operatorname{rad}(R / I)$ where $I$ is the right annihilator of $\left(a_{i}-a_{j}\right) R$ in $R$. Then $\left(b_{i} b_{j}\right)^{m} \in I$ for a positive integer $m$, depending on $(i, j)$, and $\left(a_{i}-a_{j}\right) R\left(b_{i} b_{j}\right)^{m}=$ (0). By (PS II) there exists a positive integer $k$, depending on $(i, j)$, such that $\left(a_{i}-a_{j}\right)\left(R b_{i} R\right)^{k} b_{j}^{k}=(0)$. Now since $\left(a_{i}-a_{j}\right)\left(R b_{i} R\right)^{k+1} b_{j}^{k} \subseteq\left(a_{i}-a_{j}\right)\left(R b_{i} R\right)^{k} b_{j}^{k}=$ $(0)$, we may assume that $k$ is independent of $(i, j)$. Since $\operatorname{supp} b=\operatorname{supp} R b R=$ $\operatorname{supp}(R b R)^{k}$, from $\operatorname{Spec} R=\bigcup_{i=1}^{n} \operatorname{supp} b_{i}$, we have $R=\sum_{i=1}^{n}\left(R b_{i} R\right)^{k}$. Then $1=e_{1}+e_{2}+\cdots+e_{n}$ where $e_{i} \epsilon\left(R b_{i} R\right)^{k}$. Put $a=a_{1} e_{1}+a_{2} e_{2}+\cdots+a_{n} e_{n} \cdot$ For any $s \in R, e_{i} s \in\left(R b_{i} R\right)^{k}$, hence $\left(a_{i}-a_{j}\right) e_{i} s b_{j}^{k}=0$ and $a s b_{j}^{k}=\Sigma_{i} a_{i} e_{i} s b_{j}^{k}=$ $\Sigma_{i} a_{j} e_{i} s b_{j}^{k}=a_{j} s b_{j}^{k}$, i.e., $\left(a-a_{j}\right) s b_{j}^{k}=0$. Thus $\left(a-a_{j}\right) R b_{j}^{k}=0$. By (PS I), this means that $a-a_{j} \in O(P)$ for every $P \in \operatorname{supp} b_{j}, \hat{a}(P)=\hat{a}_{j}(P)=\sigma(P)$. Hence $\sigma=\hat{a}$.

Lambek [12] calls a ring prime-torsion free if it has a prime ideal $P$ such that $O(P)$ is zero.

Theorem 3.6. If $R$ is a ring sucb that $\bigcap O(P)=0$, then $R$ satisfies (S I) iff $R$ is isomorphic onto a subring of the ring of global sections of a sheaf whose stalks are rings with (S I). In this case the stalks may be chosen to be prime-torsion free rings.

Proof. Suppose $R$ has (S I). By the proof of $2.1(\mathrm{~b})$ the stalks $R / O(P)$ satisfy (S I). $P / O(P)$ is a prime ideal of $R / O(P)$. If $a R b \subseteq O(P)$ for some $b \notin P$, then $a b^{2} \in O(P)$ and $a b^{2} R c=0$ for some $c \notin P$. By choosing $w=b u b v c \notin P$, we have $a w=0$ and $a \in O_{P}=O(P)$. Thus the ring $R / O(P)$ is prime-torsion free. Conversely, suppose $R$ is isomorphic into $\Gamma(X, k)$ for a sheaf $k=\bigcup_{x \in X} R_{x}$ over a topological space $X$, where each ring $R_{x}$ is a ring satisfying (S I). We may regard $R$ to be a subring of $\Gamma(X, k)$. Let $a, b \in R$ such that $a b=0$. For each $x \in X$, $a(x) b(x)=O_{x}$ and $a(x) R_{x} b(x)=O_{x}$ since $R_{x}$ has the property (S I). Then for any $s \in R, a(x) s(x) b(x)=O_{x}$, hence asb is the zero map, i.e., the zero element of $\Gamma(X, k)$. So we have $a R b=0$.

Corollary 3.7. If $R$ is an almost symmetric ring with identity, then $R$ is isomorphic onto $\Gamma(\operatorname{Spec} R, R(R)$ ), where $k(R)=\bigcup R / O(P)$ and each stalk is an almost symmetric, prime-torsion free ring. Conversely, if $R$ is a ring isomorphic into the ring $\Gamma(X, k)$ for a sheaf of almost symmetric rings $R_{x}$ over a compact space $X$, then $R$ is almost symmetric.

Proof. Let $R$ be an almost symmetric ring with identity. By 3.5, 2.1 and 3.6 the conclusions follow. For the converse let $R \subseteq \Gamma(X, k)$ where the stalks $R_{x}$ are almost symmetric and $X$ is compact. By 3.6, $R$ satisfies (S I). Suppose $a(b c)^{2}=0$ for some global sections $a, b, c$. For $x \in X, a(x) b(x)^{m} c(x)^{m}=0_{x}$ for a positive integer $m$. This means the section $a b^{m} c^{m}$ vanishes at $x$, hence it vanishes on a neighborhood of $x$. Since $X$ is compact there are finite number of 
open neighborhoods $N_{1}, N_{2}, \cdots, N_{n}$ and positive integers $m_{1}, m_{2}, \cdots, m_{n}$ such that $X=\bigcup_{i=1}^{n} N_{i}$ and $\left(a b^{m_{i}} c^{m_{i}}\right)(y)=O_{y}$ for every $y \in N_{i}$. Let $t$ be the largest of $m_{i}$ 's. Since each $R_{x}$ satisfies (S I), $\left(a b^{t} c^{t}\right)(y)=O_{y}$ for each $y \in N_{i}$ and each $i=1,2, \cdots, n$. Then $a b^{t} c^{t}$ must be the zero section. Thus $R$ has (S II).

For base spaces other than Spec $R$, we obtain the following:

Theorem 3.8. Let $R$ be a ring with identity such that

(i) $R$ satisfies (PS II),

(ii) $\operatorname{Max} R \subseteq X \subseteq \operatorname{Spec} R$,

(iii) for eacb a $\in R, \bigcap\left\{P \in X:(a R)^{r} \subseteq P\right\}=\left\{b \in R\right.$ : $a R b^{m}=0$ for some $\left.m\right\}$.

Then $R$ is isomorphic to the ring of global sections of a sheaf $k$ over $X$ with stalks $R / O(P)$.

Proof. Since $1 \in R$ and $\operatorname{Max} R \subseteq X, X$ is compact and $\bigcap\{O(P): P \in X\}=(0)$. If $\sigma$ is a global section then there is $a \in R$ such that $\sigma(P)=a+O(P)$ for each $P \in X$, following the proof of 3.5 .

4. Prime ideal space and compact minimal prime ideal space. Let $\operatorname{Min} R$ be the subspace of $\operatorname{Spec} R$ of all minimal prime ideals of $R$, and $\operatorname{Max} R$ be the set of all maximal ideals of $R$. If $\operatorname{Max} R \subseteq \operatorname{Spec} R$ we shall consider $\operatorname{Max} R$ to be a subspace of $\operatorname{Spec} R$. We shall adopt the notations: $s(a)=\operatorname{supp} a \cap \operatorname{Min} R, b(a)=$ hull $a \cap \operatorname{Min} R$ for any $a \in R$, and similarly for any $A \subseteq R$. The following lemma is easily proved.

Lemma 4.1. For any ring $R$ the following are equivalent:

(a) Spec $R$ is a $T_{1}$-space,

(b) $\operatorname{Spec} R=\operatorname{Min} R$.

If $\operatorname{Max} R \subseteq \operatorname{Spec} R$ and every prime ideal is contained in a maximal ideal, then the above conditions are equivalent to

(c) $\operatorname{Spec} R=\operatorname{Max} R$.

Theorem 4.2. For any ring $R$ whose prime radical is the set of all nilpotent elements, the following are equivalent:

(a) $N(P)=P$ for each $P \in \operatorname{Spec} R$,

(b) supp a is closed (as well as open) in Spec $R$ for each a $\in R$,

(c) Spec $R$ is a completely regular $T_{2}$-space,

(d) Spec $R$ is a $T_{1}$-space.

If $R$ is almost symmetric, then these are equivalent to

(e) $R / O(P)$ bas a unique prime ideal, i.e., $P / O(P)$, for each prime ideal $P$ of $R$.

Proof. (e) $\Rightarrow$ (d) always, for if $Q \in \overline{P\}}=$ hull $P$ then $P \subseteq Q$ and $O(P) \subseteq Q$.

(a) $\Rightarrow$ (b): If $a \notin P$ and $a R b \subseteq \operatorname{rad} R$ then $b \in P$. If $a \in P=N(P)$ then $a R b \subseteq \operatorname{rad} R$ 
for some $b \notin P$. Hence supp $a=$ hull $I$, where $I$ is the set of all $b \in R$ such that $a R b \subseteq \operatorname{rad} R$. (b) $\Rightarrow$ (c) $\Rightarrow$ (d) are clear. (d) $\Rightarrow$ (a) follows from 1.10 and 4.1. Now let $R$ be almost symmetric such that $\operatorname{Spec} R$ is a $T_{2}$-space. For $P, Q \in \operatorname{Spec} R$ they have disjoint open neighborhoods supp $a$ and supp $b$, respectively. Then $a b \in \operatorname{rad} R$ and $a^{m} b^{m}=0$ by (S II). There are $u \notin P, v \notin Q$ such that $u v=0$ by (S I). Thus $u \in O(Q)$ and $O(Q) \not \subset P$. Similarly $O(P) \nsubseteq \underline{Q}$ and (c) $\Rightarrow$ (e).

Gillman [3] has obtained (a) $\Leftrightarrow(b) \Rightarrow$ (c) of 4.2 in an arbitrary ring and (b) $\Leftrightarrow$ (c) in any commutative ring. For a more general version of (a) $\Leftrightarrow(b) \Leftrightarrow$ (d), see $[7,1.29$ and 1.32]. (c) $\Leftrightarrow$ (d) need not hold true in general. (See Example 5.6.)

Proposition 4.3. If $R / \mathrm{rad} R$ is a strongly regular ring with identity then Spec $R$ is a $T_{2}$-space.

Proof. Since $\operatorname{Spec} R$ is homeomorphic onto $\operatorname{Spec}(R / \operatorname{rad} R)$, we may assume that $R$ is semiprime. Let $a \notin P$ and $a \in Q$, with $a=a^{2} b$. Let $c=1-a b$. Then $a c=0, a R c=0$ since $R$ has no nilpotents, hence supp $a$ and supp $c$ are disjoint neighborhoods of $P$ and $Q$, respectively.

The simple integral domain with identity, which is not a division ring, shows that the converse of 4.3 is not true by 1.17. If $R$ is the direct product of finite number of copies of this simple integral domain, then $R$ is neither simple nor an integral domain. Evidently $\operatorname{Spec} R$ is still a $T_{2}$-space but $R$ is not regular. The following is well known for the commutative case. Recall that a ring is right (resp. left) duo if every right (resp. left) ideal is an ideal. Note that any right (or left) duo ring satisfies (S I).

Theorem 4.4. For any right (or left) duo ring with identity the following are equivalent:

(a) $R / \operatorname{rad} R$ is a (von Neumann) regular ring,

(b) Spec $R$ is a $T_{2}$-space,

(c) Spec $R$ is a $T_{1}$-space.

Proof. Let $R$ be a right duo ring. Since $R$ satisfies (S I), $R / \mathrm{rad} R$ has no nilpotents. By 4.3 it suffices to show that (c) implies (a). We may assume that $R$ is semiprime. By 4.1 every prime ideal is a maximal ideal. If $a \neq 0$, then $a \notin M$ for some $M \in \operatorname{Spec} R, M+a R=R$ and $1-a y \in M$ for some $y \in R$. \{hull $z$ : $z=1-a y$ for some $y \in R\}$ covers supp $a$ and by 4.2 this is an open covering for the compact set suppa. There are elements of the form $z_{i}=1-a y_{i}$ such that supp $a \subseteq \bigcup_{i=1}^{n}$ hull $z_{i}$, hence $a z_{1} z_{2} \cdots z_{n} \in \operatorname{rad} R=0$. But $a z_{1} z_{2} \cdots z_{n}$ is of the form $a(1-a x)$ for some $x \in R$ and then $a=a^{2} x$.

Compare 4.5 with $[7,1.27]$. 
Proposition 4.5. If every prime ideal of a ring $R$ is in a maximal ideal and $\operatorname{Max} R \subseteq \operatorname{Spec} R$, then the following are equivalent:

(a) $R / N(P)$ bas a unique maximal ideal for each $P \in \operatorname{Spec} R$,

(b) $R / N(M)$ bas a unique maximal ideal for eacb $M \in \operatorname{Max} R$.

If, in addition, $R$ is almost symmetric, then these conditions are equivalent to

(c) $R / O(P)$ bas a unique maximal ideal for each $P \in \operatorname{Spec} R$.

Proof. If $P \subseteq M$ then $N(M) \subseteq N(P) \subseteq M$ and $O(P) \subseteq N(P) \subseteq M$. This shows $(\mathrm{b}) \Rightarrow$ (a) and (c) $\Rightarrow(a)$, hence it remains to show that (a) implies (c) when $R$ is almost symmetric. For this we shall show that for each $P \in \operatorname{Spec} R, M / O(P) \epsilon$ $\operatorname{Max}(R / O(P))$ iff $M / N(P) \in \operatorname{Max}(R / N(P))$. If $M / O(P) \in \operatorname{Max}(R / O(P))$ then $M \in \operatorname{Max} R$. Suppose that $a \in N(P)$ and $a \notin M$. $a b$ is nilpotent for some $b \notin P, a^{n} b^{n}=0$ by (S II), and $u v=0$ for some $u \notin M$ and $v \notin P$ by (S I). Then $u \in O(P) \subseteq M$, a contradiction. Thus $N(P) \subseteq M$ and $M / N(P) \in \operatorname{Max}(R / N(P))$. If $M / N(P) \in \operatorname{Max}(R / N(P))$ then $M / O(P) \in \operatorname{Max}(R / O(P))$ since $O(P) \subseteq N(P)$. This completes the proof.

Proposition 4.6. Suppose the prime radical of a ring $R$ is the set of all nilpotents of $R$. If $Q \in \operatorname{Min} I$ and $I$ is an ideal of $R$, then $Q$ is also an ideal of $R$.

Proof. Since $\operatorname{rad} I=I \cap \operatorname{rad} R, \operatorname{rad} I$ is the set of all nilpotents in $I$. Let $J$ be the ideal of $R$ generated by $Q$. It suffices to show that $J \subseteq Q$. Let $x \in J$ with $x=x_{1}+x_{2}+\cdots+x_{n}$, where for each fixed $i, x_{i}$ is of the form $u+a v+v b+$ $\sum_{j=1}^{m} a_{j} v b_{j}$ such that $u, v \in Q$ and $a, b, a_{j}, b_{j} \in R$. By $1.10, u w, v z \in \operatorname{rad} I$ for some $w, z \in \backslash Q$. Then $u(w z), v b(w z), a v(w z), a_{j} v b_{j}(w z), \epsilon \operatorname{rad} R$, thus $x_{i} w z \epsilon$ $\operatorname{rad} I$ and $w z \in \Lambda \backslash$. This way we find $d_{i} \in I Q Q$ such that $x_{i} d_{i} \in \operatorname{rad} I$ and let $d=d_{1} d_{2} \cdots d_{n^{*}}$. This means that $x d \in \operatorname{rad} l$ and $d \in I \backslash Q$. Since $x \in J \subseteq I$ and $Q$ is completely prime, we have $x \in Q$.

For a more general version of 4.7 , see $[7,1.33]$.

Proposition 4.7. If the prime radical of a ring $R$ is the set of all nilpotents of $R$, then $\operatorname{Min} R$ is a $T_{2}$-space with a base of closed-and-open sets.

Proof. For $P, Q \in \operatorname{Min} R$, if $a \in P$ and $a \notin Q$, then $a b \in \operatorname{rad} R$ for some $b \notin P$ by 1.10. $s(a)$ and $s(b)$ are the required disjoint open sets. For each $a \in R, s(a)=$ $b(I)$, where $I$ is the set of all $b \in R$ such that $a b \in \operatorname{rad} R$. This completes the proof.

Corollary 4.8. Suppose the prime radical of a ring $R$ is the set of all nilpotent elements. If $\operatorname{Min} R$ is compact, then for each $a \in R$ there is a finitely generated ideal $I$ of $R$ such that $s(a)=b(I)$.

Proof. For each $a \in R, b(a)$ is compact and open, hence $b(a)=\bigcup_{i=1}^{n} s\left(b_{i}\right)=$ $s(l)$ where $I$ is the ideal of $R$ generated by $b_{i}$ 's.

All the results of Henriksen and Jerison [6] on minimal prime ideals of a 
commutative ring are true for the ir counterpart in a ring whose prime radical is the set of all nilpotents. The following three theorems are listed here without proofs as the proofs in [6] need only minor modifications for our case. Since $\operatorname{Min} R$ is homeomorphic onto $\operatorname{Min}(R / \operatorname{rad} R)$, we may assume that $\operatorname{rad} R=0$, i.e., that $R$ has no nilpotents, in considering the minimal prime ideal space.

Theorem 4.9. Let $R$ be a ring without nilpotents. Then the following are equivalent:

(a) Min $R$ is compact and, for any $a, b \in R, a^{r} \cap b^{r}=c^{r}$ for some $c \in R$,

(b) Min $R$ is compact and $\{b(a): a \in R\}$ is a base (for open sets) for Min $R$,

(c) for eacb a $\in R, a^{r}=\left(b^{r}\right)^{r}$ for some $b \in R$.

Theorem 4.10. Let $R$ be a ring without nilpotents. Then the following are equivalent:

(a) $\operatorname{Min} R$ is compact, extremally disconnected and, for eacb $a, b \in R, a^{r} \cap$ $b^{r}=c^{r}$ for some $c \in R$,

(b) for any subset $B \subseteq R, B^{r}=y^{r}$ for some $y \in R$.

Theorem 4.11. Let $R$ be a ring without nilpotents sucb that $\operatorname{Min} R$ is compact. If, for each sequence $\left\{a_{i}\right\}$ of elements of $R, \bigcap_{i=1}^{\infty} a_{i}^{r}=b^{r}$ for some $b \in R$, then $\operatorname{Min} R$ is basically disconnected.

Mewborn [13] has obtained a characterization of a commutative ring with identity whose minimal prime ideal space is compact, generalizing the result due to Henriksen and Jerison [6]. Our aim here is to obtain a similar characterization for the noncommutative case. A ring $T$ is a right quotient ring of a subring $R$ provided $T_{R}$ is a rational extension of $R_{R}$. If $R$ is a subring of $T$, we shall use the notation: $S(a)=\{M \in \operatorname{Spec} T: a \notin M\}, H(a)=\operatorname{Spec} T \backslash S(a)$ for each $a \in T$. Also recall that for each $a \in R, s(a)=\operatorname{supp} a \cap \operatorname{Min} R$ and $b(a)=\operatorname{Min} R \backslash s(a)$.

Lemma 4.12. If $R$ is a subring of $T$ then for eacb $P \in \operatorname{Spec} R$ there is a $M \in \operatorname{Spec} T$ sucb that $M \cap R \subseteq P$.

Proof. $R \backslash P$ is an $m$-system in $T$, disjoint from (0).

Theorem 4.13. Suppose a ring $R$ bas a right quotient ring $T$ which bas no nilpotents. If Spec $T$ is a compact $T_{1}$-space then the following are equivalent:

(a) $\operatorname{Min} R$ is compact,

(b) $\operatorname{Min} R=Y$, where $Y=\{M \cap R: M \in \operatorname{Spec} T\}$,

(c) for each a $\in R, S(a)=H(I)$ for a finitely generated ideal I of $R$.

If, in addition to conditions on $T, R$ bas an identity and $T_{R}$ is a flat module, then $\operatorname{Min} R$ is compact.

Proof. Note that $\operatorname{Spec} T=\operatorname{Min} T$ by 4.1 and each $M \in \operatorname{Spec} T$ is completely 
prime by 1.11, thus $M \cap R$ is completely prime in $R$ if $M \cap R \neq R$. By Lemma 4.12 we have $\operatorname{Min} R \subseteq Y$. (b) $\Rightarrow$ (a): Since $\operatorname{Min} R=Y$ the map $f: \operatorname{Spec} T \rightarrow \operatorname{Spec} R$ defined as $f(M)=M \cap R$ is continuous and $\operatorname{Min} R$ is compact as it is the image of a compact space by a continuous map.

(a) $\Rightarrow$ (b). Suppose that $M_{0} \cap R \notin \operatorname{Min} R$ for some $M_{0} \in \operatorname{Spec} T$. $M_{0} \cap R \nsubseteq P$ for each $P \in \operatorname{Min} R$. Since $\operatorname{Min} R$ is compact, there are elements $a_{i} \in M_{0} \cap R$ such that $\operatorname{Min} R=\bigcup_{i=1}^{n} s\left(a_{i}\right)$. Let $I$ be the ideal of $R$ generated by $a_{i}$ 's. Then $S(I)=\bigcup_{i=1}^{n} S\left(a_{i}\right)$ is closed in Spec $T$ by 4.2. Hence $M_{0} \in S(\alpha) \subseteq H(I)$ for some $\alpha \in T$. Then $\alpha \neq 0, l \alpha=0$ and the right annihilator of $l$ in $T$ is a nonzero right ideal, therefore $I c=0$ for some $0 \neq c \in R$ since $T_{R}$ is an essential extension of $R_{R}$. Hence $S(c) \subseteq H(I)$. Since $c \notin \operatorname{rad} R=0$, there is $P \in s(c)$ and $M \cap R=P$ for some $M \in \operatorname{Spec} T$ by 4.12. Then $M \in S(c) \subseteq H(I)$ and $P \in b(I)$, which is impossible since $s(I)=\operatorname{Min} R$. This shows that $Y \subseteq \operatorname{Min} R$, hence $Y=\operatorname{Min} R$. (b) $\Rightarrow$ (c). Let $a \in R$. If $H(a)=\varnothing$ let $I=(0)$. Let $M_{0} \in H(a)$. Since $Y=\operatorname{Min} R, M \cap$ $R \nsubseteq M_{0} \cap R$ for any $M \in S(a)$. $S(a)$ is closed and compact, $S(a) \subseteq \bigcup\{H(b)$ : $\left.b \in R \backslash M_{0}\right\}$, hence $S(a) \subseteq \bigcup_{i=1}^{n} H\left(b_{i}\right)=H(c)$ with $c \in R \backslash M_{0}$. If $S(a)=\varnothing$ choose any $c \in R \backslash M_{0^{*}}$. Then $M_{0} \in S(c) \subseteq H(a)$ and $H(a)=\bigcup_{j=1}^{m} S\left(c_{j}\right)=S(I)$ where $I$ is the ideal of $R$ generated by $c_{j}$ 's. (c) $\Rightarrow$ (b). Suppose $M_{0} \cap R \notin \operatorname{Min} R$ for a $M_{0} \in \operatorname{Spec} T$. Either $M_{0} \cap R=R$ or $M_{0} \cap R \in \operatorname{Spec} R$. Note that $\operatorname{Min} R \neq \varnothing$ since $\operatorname{rad} R=0$. There is $M_{1} \in \operatorname{Spec} T$ such that $M_{1} \cap R \subsetneq M_{0} \cap R$ since $\operatorname{Min} R \subseteq Y$. Let $a \in M_{0} \cap R$ and $a \notin M_{1}$. By (c), $S(a)=H(I)$ for an ideal $I$ of $R$. Then $I \subseteq M_{1} \cap$ $R \subsetneq M_{0}$ and $M_{0} \in H(I)=S(a)$, a contradiction. Now suppose that $R$ has an identity and $T_{R}$ is flat. By 3.5, $T \cong \Gamma(\operatorname{Spec} T, \bigcup T / O(M))$ and $O(M)=M$ for each $M \in \operatorname{Spec} T$ by 4.2. Let $a \in R$. Since $S(a)$ is closed and open in Spec $T$, we have $e \in T$ when $e: \operatorname{Spec} T \rightarrow \bigcup T / O(M)$ is defined by $e(M)=0+M$ for each $M \in S(a)$ and $e(M)=1+M$ for each $M \in H(a)$. Since $e a=0$ and $T_{R}$ is flat, there are $y_{j} \in T$ and $b_{j} \in R$ such that $e=\Sigma_{j=1}^{m} y_{j} b_{j}$ and $b_{j} a=0$ for each $j=1,2, \cdots, m$ by [1, Exercise VI-6]. Let $I$ be the ideal generated by $b_{j}$ 's. Then $H(a)=S(e) \subseteq S(I)=$ $\bigcup_{j=1}^{m} S\left(b_{j}\right) \subseteq H(a)$. This proves the theorem.

Proposition 4.14 (Koh). If a ring $R$ bas no nilpotents then its right and left singular ideals are zero.

Proof. See $[9,2.6]$.

Proposition 4.15. If $R$ is a symmetric ring with identity, then its right singular ideal coincides with its left singular ideal.

Proof. Let $Z_{r}(R)$ be the right singular ideal of $R$ and let $a \in Z_{r}(R)$. Suppose $I$ is a left ideal of $R$ such that $a^{l} \cap I=(0)$. For each $b \in I$ and for any $b c \in a^{r} \cap$ $b R, a b c=0$ and $c b a=0$ since $1 \in R, c b \in a^{l} \cap I=(0)$ and $b c=0$. Then $a^{r} \cap b R=0$ and $b=0$, hence $l=0$, showing that $a \in Z_{l}(R)$. Similarly $Z_{l}(R) \subseteq Z_{r}(R)$. 
The singular ideals of a symmetric ring, of course, need not be zero as the ring of integers modulo four indicates. In an almost symmetric ring, the right and the left singular ideals need not coincide even if it has an identity. (See 5.1(d).)

Proposition 4.16. Let $R$ be a ring with identity. If $P \in \operatorname{Spec} R$ and $I_{R}$ is the injective bull of the right $R-\operatorname{module} R / P$, then $O(P) \subseteq I^{r} \subseteq O_{P}$, where $I^{r}$ is the right annibilator of $I$ in $R$. If $R$ satisfies $(S I)$ then $O(P)=I^{r}$.

Proof. Let $a \in O(P)$ with $a R b=0$ for some $b \notin P$. If $a \in I$ then $a a R \cap R / P$ is a submodule of $R / P$, hence is equal to $J / P$ for a right ideal $J$ of $R$. $(J / P) b \subseteq a a R b=0, J b \subseteq P$ and $J \subseteq P$, thus $J=P$. This means $a a R=0$ and $a a=0$. Now suppose $a \notin O_{P^{\circ}}$. Then $a^{r} \subseteq P$ and $(R / P) a^{r}=0$. For $a \in R / P$ define $f_{a}: a R \rightarrow I_{R}$ by $f_{a}(a r)=a r$. Let $g_{a}: R_{R} \rightarrow I_{R}$ be the extension of $f_{\alpha}$. Now $a=f_{\alpha}(a)=g_{\alpha}(a)=g_{\alpha}(1) a \in I a$, showing that $R / P \subseteq l a$ and $l a \neq 0$.

The proof above is an adaptation from $[9,2.81$.

Proposition 4.17 (Koh). For any ring $R$ the following are equivalent:

(a) The injective bull $\hat{R}$ of $R_{R}$ is a strongly regular ring.

(b) $Z_{r}(R)=0$ and $m(R)=1$, where $m(R)$ is the least upper bound of integers $n$ such that $R$ contains a direct sum of $n$ mutually isomorphic nonzero right ideals of $R$.

Proof. See [10, 4.37].

Remarks 4.18. Concerning the hypotheses of 4.13 we note the following:

(a) If $T$ is a right quotient ring of a semiprime ring $R$, then $T$ is also semiprime since $R \cap \operatorname{rad} T \subseteq \operatorname{rad} R$ by 4.12 .

(b) If $R$ has no nilpotents then by $4.14 \hat{R}$, the injective hull of $R_{R}$, is the maximal right quotient ring of $R$ and it is a (von Neumann) regular ring with identity. But $\hat{R}$ may have nilpotents $[9,2.7]$.

(c) If $R$ has no nilpotents such that $m(R)=1$, then by $4.17 \hat{R}$ is strongly regular. Hence $\hat{R}$ has no nilpotents and Spec $\hat{R}$ is a compact $T_{1}$-space by 4.3 . Therefore we may choose in this case $T=\hat{R}$ for 4.13 .

(d) Spec $R$ of 4.13 need not be a $T_{1}$-space. Let $R$ be the ring of integers and $T$ the rationals.

(e) The right quotient ring $T$ of 4.13 need not be a regular ring. Let $T=R$ be the simple integral domain with identity which is not a division ring.

(f) Spec $T$ of 4.13 need not be a finite set. Let $R=T=C(N)$ the ring of real-valued continuous functions on the space of natural numbers. The cardinality of Spec $T=\operatorname{Max} T=\beta N$ is $2^{c}[4,9.3]$.

5. Examples and counterexamples.

Example 5.1. For a ring $S$ with identity, let $R=\left\{a_{0}+a_{1} x+a_{2} y+a_{3} z: a_{i} \in S\right\}$ 
with indeterminates $x, y$ and $z$. By defining multiplication as

$$
\begin{aligned}
\left(a_{0}+a_{1} x+\right. & \left.a_{2} y+a_{3} z\right)\left(b_{0}+b_{1} x+b_{2} y+b_{3} z\right) \\
& =a_{0} b_{0}+\left(a_{0} b_{1}+a_{1} b_{0}\right) x+\left(a_{0} b_{2}+a_{2} b_{0}\right) y+\left(a_{0} b_{3}+a_{3} b_{0}+a_{1} b_{2}\right) z
\end{aligned}
$$

$R$ becomes a ring with identity with the usual addition. [ $R$ may be described in a different manner (see Example 5.2 also): Let $T$ be the free module over integers $Z$ with bases $\{1, x, y, z\}$. Define a multiplication on $T$ such that all products are zero except that $x y=z$ and that 1 acts as an identity. Then let $R=S \otimes_{Z} T_{\text {.] }}$

(a) (i) $S$ has no nilpotents iff $R$ satisfies (S I).

(ii) If $S$ has no nilpotents, then $u(v w)^{2}=0$ implies $u v^{2} w^{2}=0$ for any $u$, $v, w \in R$.

(iii) $R$ is not symmetric.

Proof. If $a^{n}=0$ for some $a \in S$ then $\left(a^{n-1}+x\right)\left(a^{n-1}-x\right)=0$, but $\left(a^{n-1}+x\right) y\left(a^{n-1}-x\right)=a^{n-1} z$. If $S$ has no nilpotents one can show, through direct computation, that $R$ satisfies (S I) and that $u(v w)^{2}=0$ implies $u v^{2} w^{2}=0$ for any $u, v, w \in R . R$ is not symmetric since $1 y x=0$ and $1 x y=z$.

(b) Spec $R$ is homeomorphic onto Spec $S$.

Proof. Note that the ideal $(x, y, z)=x R+y R$, which is the set of all elements of $R$ with zero constant terms, is nilpotent with nilpotency 3. $x R+y R \subseteq \operatorname{rad} R$. For $P \in \operatorname{Spec} R$ let $\phi(P)=\{a \in S: a \in P\}$. Then $\phi$ is the required homeomorphism.

(c) Let $S=Z /(4)$ the ring of integers modulo four. Then

(i) $R$ satisfies (S II) but not (S I),

(ii) $R$ is pseudo symmetric.

Proof. (i) Spec $R=\{P\}$ by (b) where $P$ is the set of elements whose constant term is in (2)/(4). Let $u(v w)^{n}=0$. If $v \in P$ or $w \in P$ then $u v^{4} w^{4}=0$ since $P^{4}=0$. If $u \notin P$ and $v \notin P$ then $u \in O_{P}$ since $P$ is completely prime. But $O_{P}=0$. Hence $R$ satisfies (S II). (ii) $R$ satisfies (PS I) by 1.13 . (PS II) holds by the same argument as for (S II) in (i) above.

(d) Let $S$ be the ring of integers. Then $Z_{r}(R) \neq Z_{l}(R)$.

Proof. By (a) $R$ is almost symmetric. $x^{l}=\operatorname{rad} R$ is the set of all elements with zero constant terms. If $x^{l} \cap l=(0)$ for a left ideal $l$ and $a=a_{0}+a_{1} x+$ $a_{2} y+a_{3} z \in I$, then $z a=a_{0} z \in x^{l} \cap I$ and $a_{0}=0$. Hence $a \in x^{l}$ and $I=(0)$, showing that $x \in Z_{l}(R)$. Now for any $y a \in x^{r} \cap y R, 0=x y a=z a=a_{0} z$ and $a_{0}=0$. Then $y a=0$ and $x \notin Z_{r}(R)$. Similarly $y \in Z_{r}(R)$ and $y \notin Z_{l}(R)$.

In $[7$, p. 311] Hofmann asks whether there is a ring with identity, outside the commutative case, outside the strongly harmonic case, and outside the semiprime rings, which is isomorphic to the ring of global sections of a sheaf over the maximal ideal space. The following example is such a one: 
(e) Let $S$ be the ring of integers. Then $R$ is not commutative. Koh [11] calls a ring strongly barmonic iff for any two maximal modular ideals $M \neq N$, there are ideals $I, J$ such that $I \nsubseteq \mathbb{M}, J \underline{\&} N$, and $I J=0$. Now for any $P, Q \in \operatorname{Spec} R$, if $a \notin P, b \notin Q$ then $a b \neq 0$ since $a_{0} b_{0} \neq 0$. Hence this ring $R$ is not strongly harmonic. $R$ is not semiprime since $\operatorname{rad} R=x R+y R$. Note that $O(M)=\breve{M}=(0)$ for each $M \in \operatorname{Max} R$. By 3.8 with $X=\operatorname{Max} R, R$ is isomorphic to the ring of global sections of a sheaf over Max $R$ with stalks $R / O(M)=R / \breve{M}=R$.

Example 5.2. Let $S$ be a ring without nilpotents and with an identity. Let $S[x, y]$ be the free ring generated by indeterminates $x$ and $y$ over $S$. For each positive integer $a$, let $R_{a}=S[x, y] /\left(x^{a+1}, y^{a+1}, y x\right)$. Note that the ring of Example 5.1 is the case when $\alpha=1$, by identifying $x y$ with $z$. Let $R=\Pi_{a=1}^{\infty} R_{\alpha}$.

(a) By similar calculations as for 5.1 one can show that each $R_{a}$ is almost symmetric.

(b) By 2.3. (a) $R$ satisfies (S I). Let $a, b \in R$ such that $a=(x, x, x, \cdots)$ and $b=(y, y, y, \cdots) .1(a b)^{2}=0$ but $1 a^{m} b^{m} \neq 0$ for any positive integer $m$. Thus $R$ does not satisfy (S II).

(c) By $1.6 R$ satisfies (PS I) but not (PS II).

Example 5.3. This appears in another context in [5].

Let $F$ be the field of rational functions in $y$ over $Z /(2)$. Let $R$ be the polynomial ring over $F$ in an indeterminate $x$, subject to $x y+y x=1$. Then $R$ is an integral domain and $M=x^{2} R$ is a maximal two-sided ideal of $R$. $M$ is not completely prime and $R / M$ does not satisfy (PS I).

Example 5.4. For a ring $S$, let $R=\left[\begin{array}{ll}S & S \\ 0 & 0\end{array}\right]$.

(a) $R$ has ( $\mathrm{S} \mathrm{I}$ ) iff $S$ has ( $\mathrm{I} \mathrm{I}$ ). If $S$ is symmetric then $R$ is almost symmetric. $R$ is never symmetric.

(b) If $0 \neq e=e^{2} \in S$ then $\left[\begin{array}{ll}e & 0 \\ 0 & 0\end{array}\right]$ is a noncentral idempotent. Of course $R$ does not have an identity.

(c) Let $S=Z /(2)$. The only nonzero right ideals of $R$ are $R$ and $\left[\begin{array}{ll}0 & S \\ 0 & 0\end{array}\right]$, and $R$ is right duo. But $R$ is not symmetric.

(d) Let $S=Z /(2)$, and let $R^{*}$ be the ring obtained by adjoining an identity to $R$ in the usual manner. $\left(\left[\begin{array}{ll}1 & 0 \\ 0 & 0\end{array}\right], 1\right)$ is a noncentral idempotent of $R^{*}$. By $2.7, R^{*}$ does not satisfy (S I).

Example 5.5. Let $R$ be the ring of matrices over integers of the form $\left[\begin{array}{ll}a & b \\ c & d\end{array}\right]$ such that $a+d, b$ and $c$ are even integers. Only idempotents of $R$ are the zero and the identity. But $R$ does not satisfy (S I) for $\left[\begin{array}{ll}0 & 0 \\ 2 & 0\end{array}\right]\left[\begin{array}{ll}0 & 0 \\ 2 & 0\end{array}\right]=\left[\begin{array}{ll}0 & 0 \\ 0 & 0\end{array}\right]$ and $\left[\begin{array}{ll}0 & 0 \\ 2 & 0\end{array}\right]\left[\begin{array}{ll}0 & 2 \\ 0 & 0\end{array}\right]\left[\begin{array}{ll}0 & 0 \\ 2 & 0\end{array}\right]=\left[\begin{array}{ll}0 & 0 \\ 8 & 0\end{array}\right]$.

Example 5.6. Let $R$ be the ring of all sequences $a=\left\{a_{n}\right\}$ of $2 \times 2$ matrices $a_{n}$ over a division ring $D$, each of the sequence $a=\left\{a_{n}\right\}$ having an integer $N(a)$ and a diagonal matrix $d(a)$ such that $a_{n}=d(a)$ for all $n>N(a)$. For each $n$, let $I_{n}$ 
be the ideal of all sequences with zero $n$th term, and let $U$ (resp. $L$ ) be the ideal of all sequences $a$ such that the upper (resp. lower) diagonal entry of $d(a)$ is zero. Then $R / U$ and $R / L$ are isomorphic to $D$ and each $R / I_{n}$ is isomorphic to the simple ring of $2 \times 2$ matrices over $D$, hence the ideals $U, L$, and $I_{n}$ are maximal ideals. Moreover these are all the nontrivial ideals of $R$. By 4.1(c), Spec $R$ is a $T_{1}$-space. Spec $R$ cannot be $T_{2}$ since the sequence $\left\{I_{n}\right\}$ converges to both $U$ and $L$. Note that $R$ is (von Neumann) regular, hence is semiprime. Note also that $O(U)=N(U)=U \cap L \neq U$. (See Theorem 4.2.)

\section{REFERENCES}

1. H. Cartan and S. Eilenberg, Homological algebra, Princeton Univ. Press, Princeton, N. J., 1956. MR 17, 1040.

2. J. Dauns and K. H. Hofmann, Representation of rings by sections, Mem. Amer. Math. Soc. No. 83 (1968). MR $40 \# 752$.

3. L. Gillman, Rings with Hausdorff structure space, Fund. Math. 45 (1957), 1-16. MR 19, 1156.

4. L. Gillman and M. Jerison, Rings of continuous functions, University Series in Higher Math., Van Nostrand, Princeton, N. J., 1960. MR 22 \#6994.

5. W. D. Gwynne and J. C. Robson, Completions of non-commutative Dedekind prime rings, J. London Math. Soc. 4 (1972), 346-352.

6. M. Henriksen and M. Jerison, The space of minimal prime ideals of a commutative ring, Trans. Amer. Math. Soc. 115 (1965), 110-130. MR 33 \#3086.

7. K. H. Hofmann, Representations of algebras by continuous sections, Bull. Amer. Math. Soc. 78 (1972), 291-373.

8. J. Kist, Minimal prime ideals in commutative semigroups, Proc. London Math.

Soc. (3) 13 (1963), 31-50. MR $26 \# 1387$.

9. Kwangil Koh, On functional representations of a ring without nilpotent elements,

Canad. Math. Bull. 14 (1971), 349-352.

10. - Quasisimple modules and other topics in ring theory, Lecture Notes in Math., vol. 246, Springer-Verlag, New York, 1972.

1.1. - On a representation of a strongly harmonic ring by sheaves, Pacific J. Math. 41 (1972), 459-468.

12. J. Lambek, On the representations of modules by sheaves of factor modules, Canad. Math. Bull. 14 (1971), 359-368.

13. A. C. Mewborn, Some conditions on commutative semiprime rings, J. Algebra 13 (1969), 422-431. MR $43 \# 1957$.

14. R. S. Pierce, Modules over commutative regular rings, Mem. Amer. Math. Soc. No. 70 (1967). MR $36 \# 151$.

DEPARTMENT OF MATHEMATICS, NORTH CAROLINA STATE UNIVERSITY, RALEIGH, NORTH CAROLINA 27607

Current address: SINGER Simulation Products Division, 11800 Tech Road, Silver Spring, Maryland 20904 